\title{
Machine Learned Charge Transfer Integrals for Multiscale Simulations in Organic Thin Films
}

Michael Rinderle, ${ }^{\dagger}$ Waldemar Kaiser, ${ }^{\dagger}$ Alessandro Mattoni, ${ }^{\dagger}$ and Alessio Gagliardi, ${ }^{*} \dagger$

$\dagger$ Department of Electrical and Computer Engineering, Technical University of Munich, Karlstraße 45, 80333 Munich, Germany

¥Istituto Officina dei Materiali, CNR-IOM SLACS Cagliari, Cittadella Universitaria, Monserrato (CA) 09042-I, Italy

E-mail: alessio.gagliardi@tum.de 


\section{A Structure of MD Dataset}
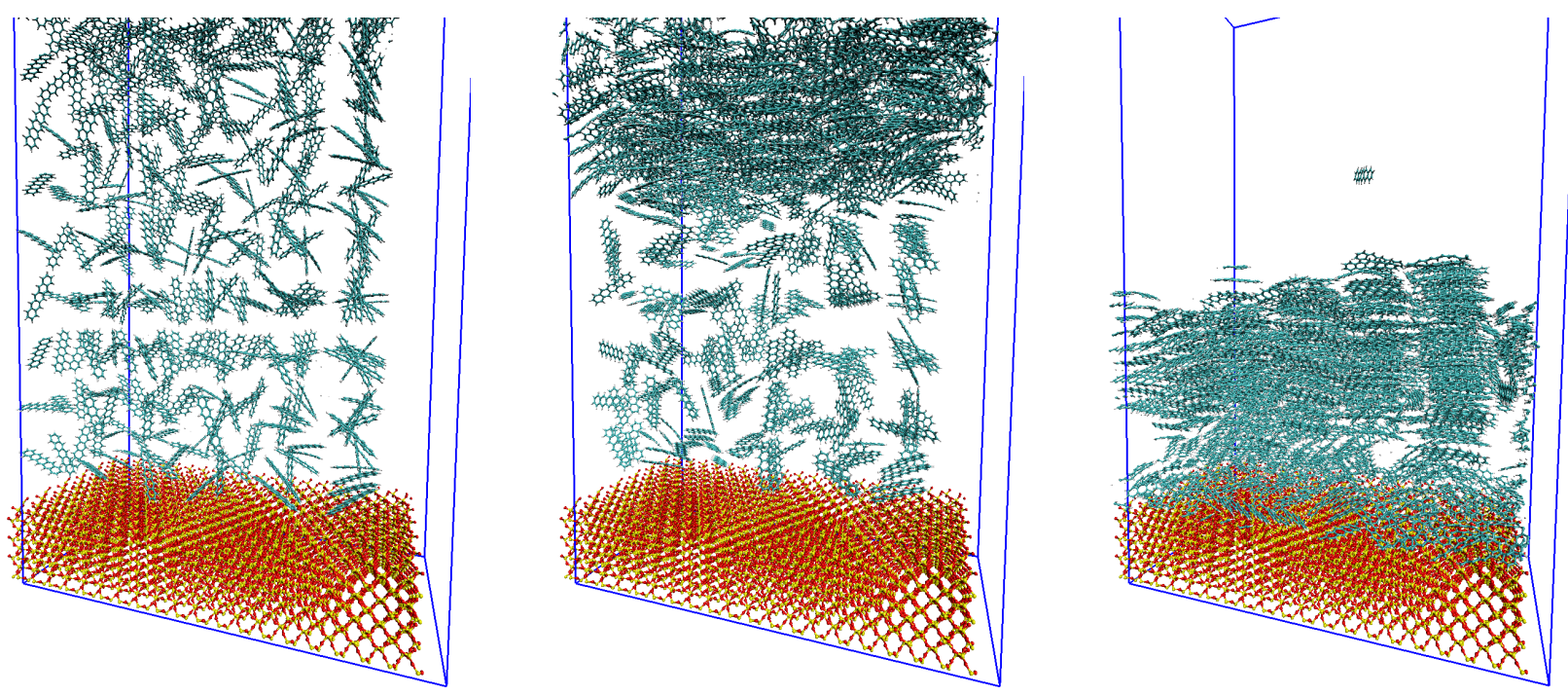

Figure S1: MD snapshots of the pentacene thin film on top of a $\mathrm{SiO}_{2}$ substrate: (left) initial structure, (mid) after 20 ps, (right) after 200 ps.

Figure S1 shows the system evolution during the Molecular Dynamics from the initial distribution (left) to the final distribution (right). The dataset used for training machine learning models is extracted from MD simulations. We extracted a set of 15000 dimers using the Voronoi tesselation and filtered them with a cut-off distance of $d \leq 18 \AA$. In Figure S2 the spatial distribution of the dimers is shown. Figure S2(a) shows a histogram of the radial distribution and the azimuthal angle distribution (the in plane angle). Many dimers show short distances (around $5 \AA$ ), an azimuthal angle close to $90^{\circ}$, and a polar angle of around $25^{\circ}$. This indicates some degree of crystallinity within our morphology. Another accumulation can be seen close to $d=16 \AA$ and small angles, which relates to the weak transport direction of a pentacene crystal. This is the reason why we chose a cut-off above this distance.

The distribution of our prediction target $V_{i j}$ is shown in Figure S3. The dataset spans close to 20 orders of magnitude in $\left|V_{i j}\right|$. In Figure $\mathrm{S} 3(\mathrm{~b})$ we show $\left|V_{i j}\right|$ versus the spatial distribution. It is clearly visible, that the highest transfer integrals are distributed along the main transport direction of a pentacene crystal. It can be seen that $\left|V_{i j}\right|$ drops with distance, but for a dimer along the weak 
(a)

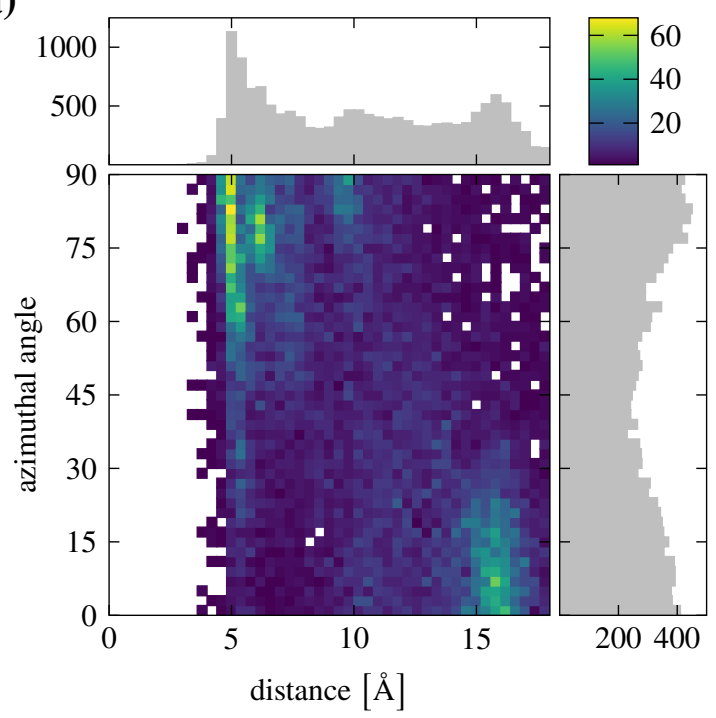

(c)

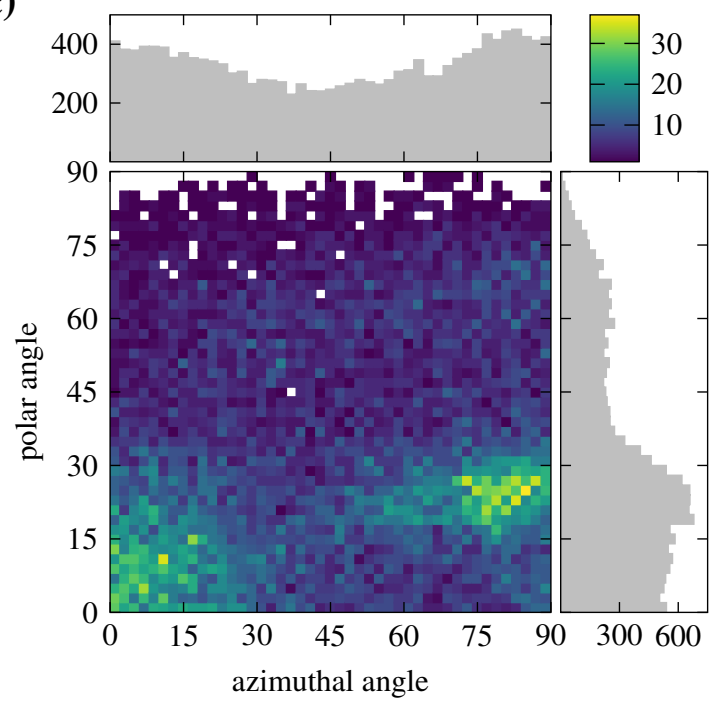

(b)

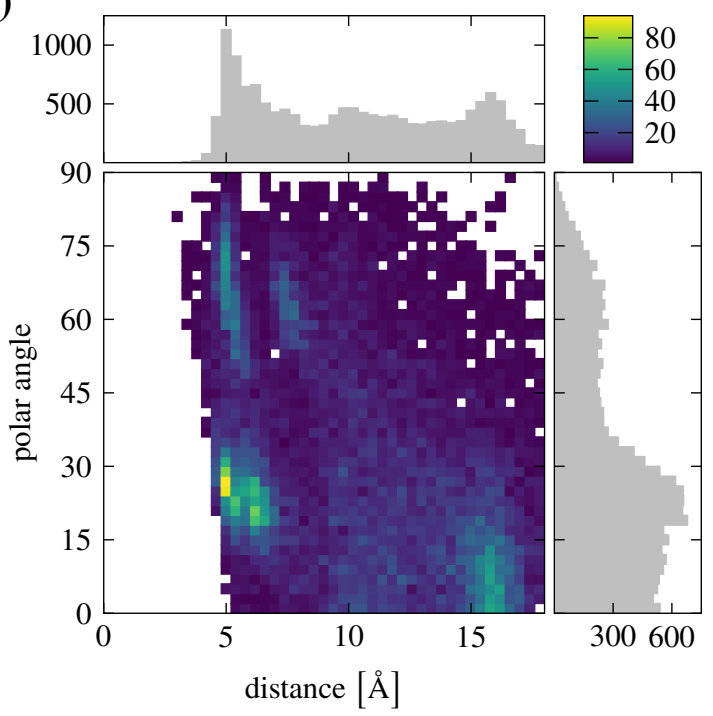

(d)

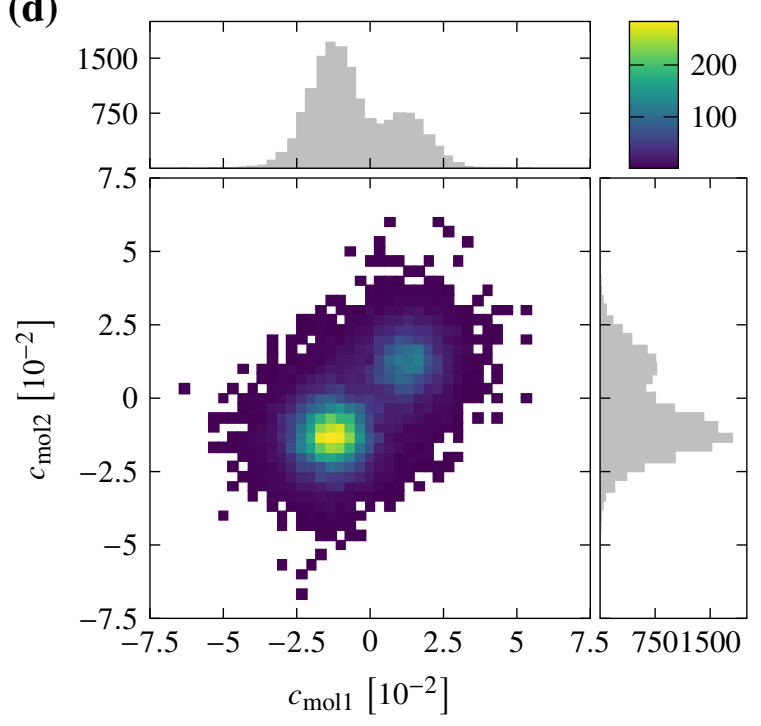

Figure S2: Spatial distribution of the dimers in the MD15k dataset. (a) distance and azimuthal angle distribution, (b) distance and polar angle distribution, (c) polar vs. azimuthal angle distribution, and (c) curvature distribution of the two molecules. 
transport direction of a pentacene crystal it is still many orders of magnitude higher than for dimers in other directions.
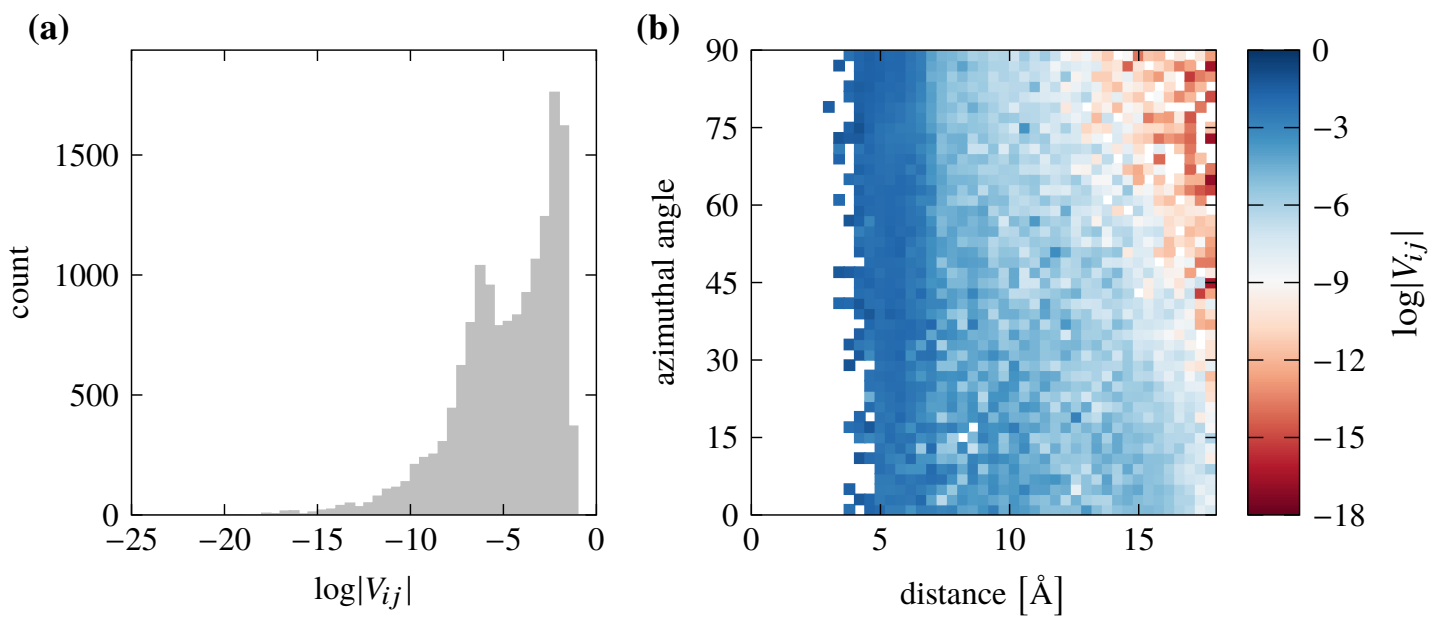

Figure S3: Distribution of the transfer integrals $V_{i j}$ within the MD15k dataset. (a) histogram of $V_{i j}$ showing the dataset spans close to 20 orders of magnitude. (b) Magnitude of transfer integrals linked to spatial distribution.
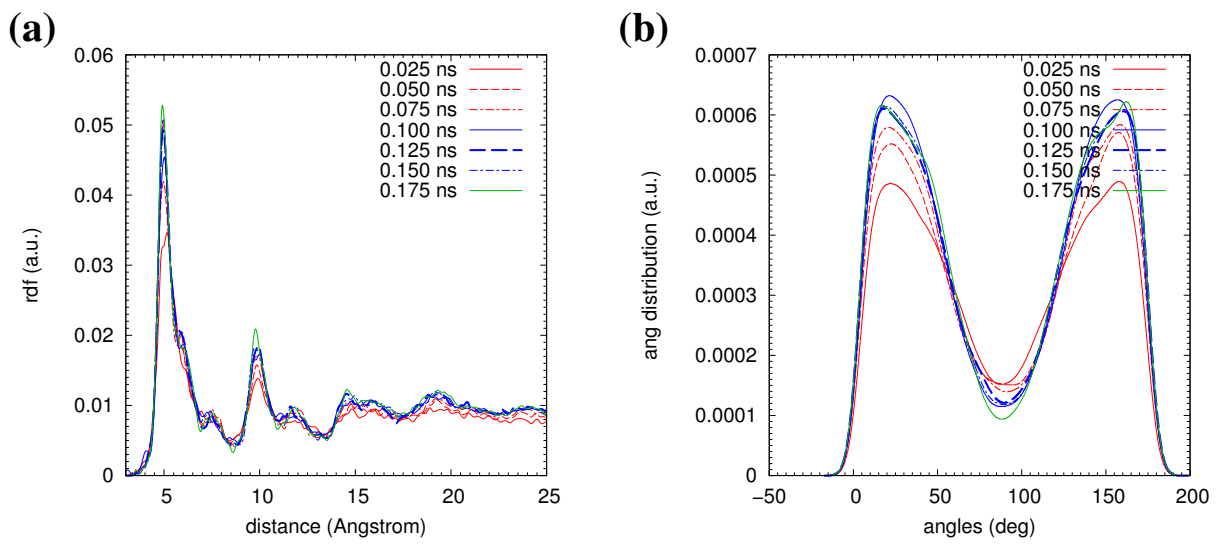

Figure S4: The pair correlation function (a) and the angular pair correlation function (b) are calculated from the pentacene geometries (centers and relative orientations) taken at different times during annealing (see legend). The peaks become more sharp during annealing indicating an increasing molecular order. 


\section{B Structure of Artificial Dataset}

(a)

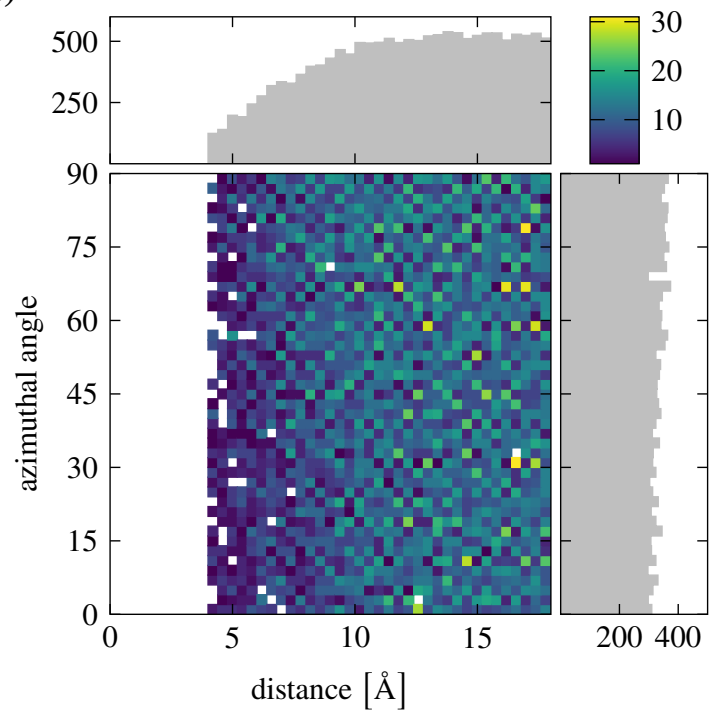

(c)

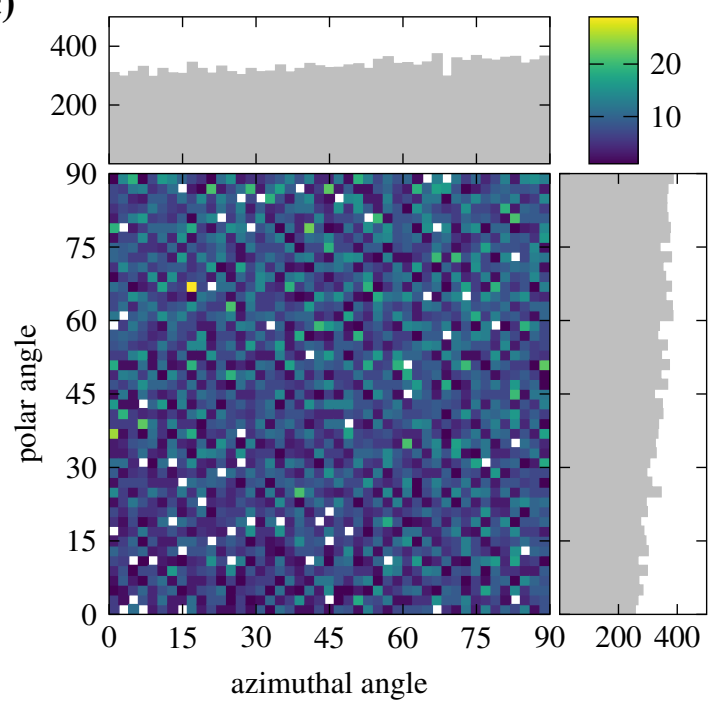

(b)

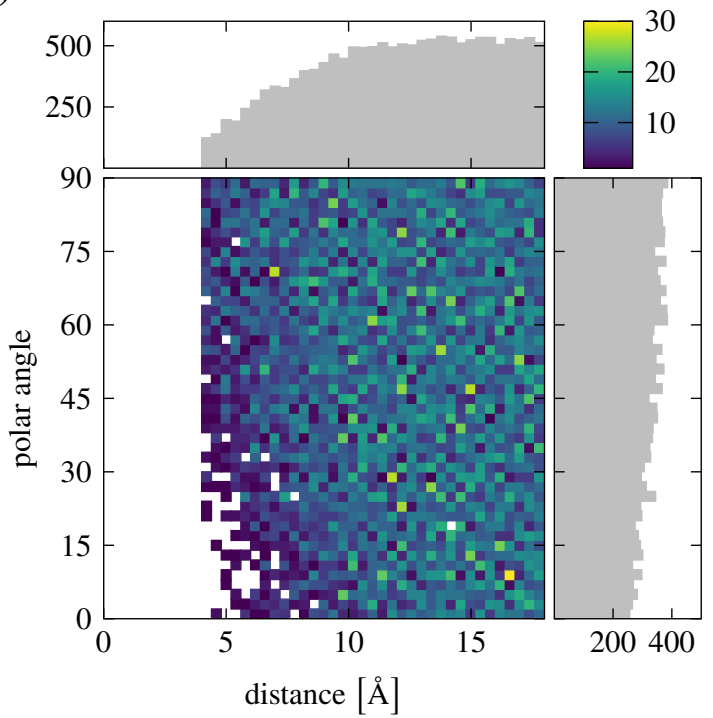

(d)

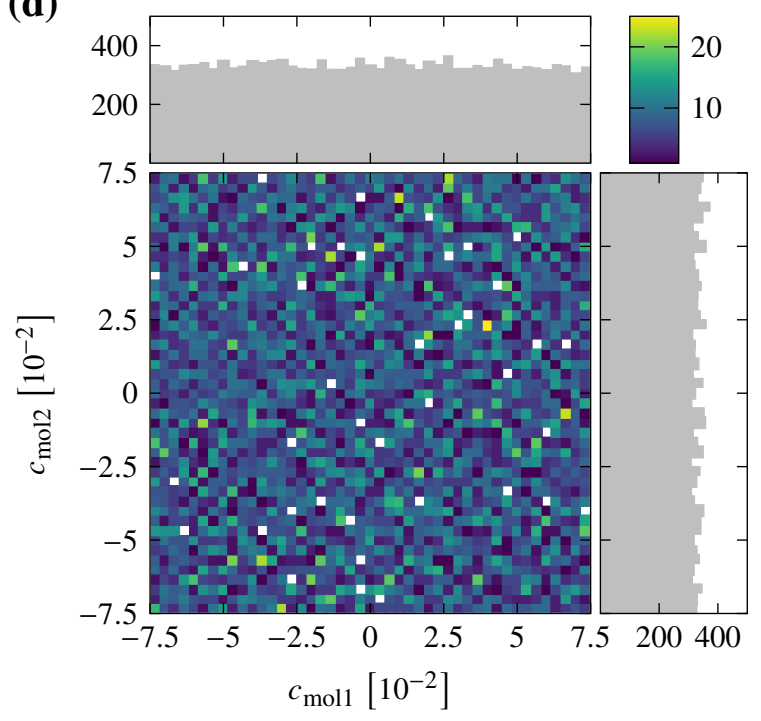

Figure S5: Spatial distribution of the dimers in the sampled dataset SP15k. (a) distance and azimuthal angle distribution, (b) distance and polar angle distribution, (c) polar vs. azimuthal angle distribution, and (c) curvature distribution of the two molecules. 

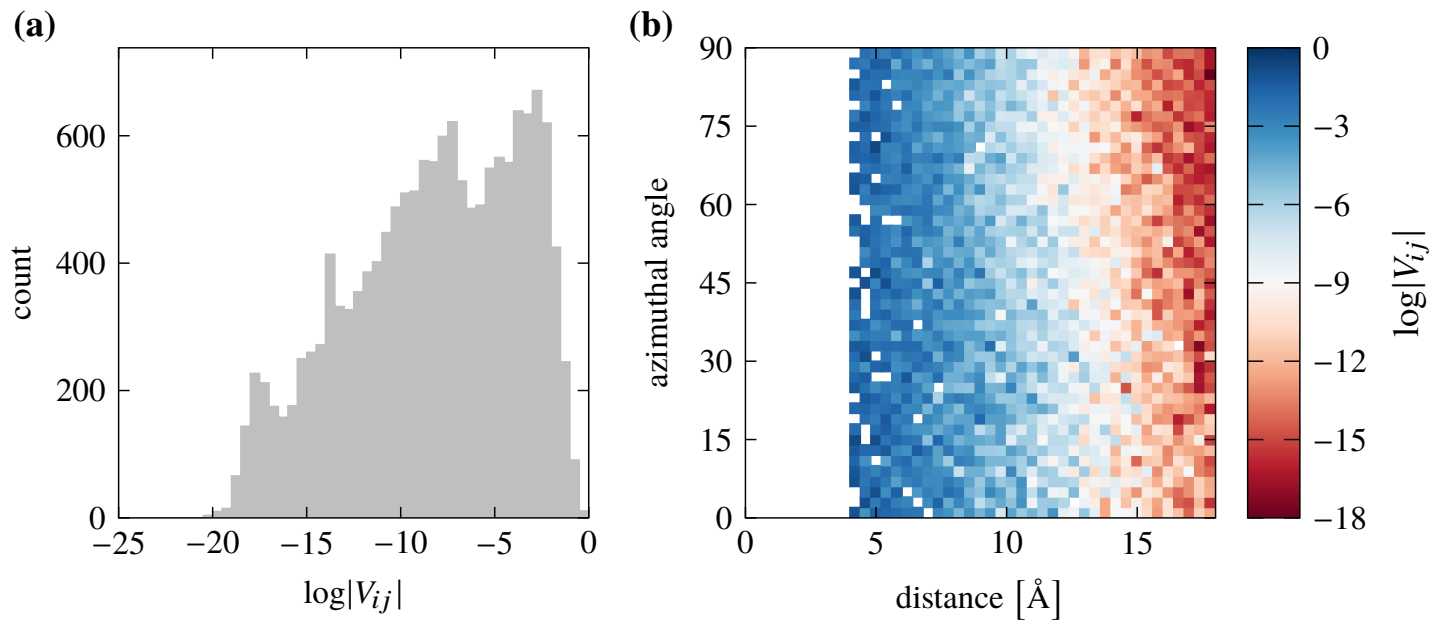

Figure S6: Distribution of the transfer integrals $V_{i j}$ within the sampled dataset SP15k. (a) histogram of $V_{i j}$. (b) Magnitude of transfer integrals linked to spatial distribution. 


\section{Machine Learning}

Table S1: List of optimized hyper-parameters used for the ML models. The regularization constant $\lambda$ is used in KRR models and $C$ is used in SVR models. The kernel width of both Gaussian and Laplacian kernels is $\gamma$.

\begin{tabular}{lccc}
\hline & $\lambda$ & $\boldsymbol{C}$ & $\boldsymbol{\gamma}$ \\
\hline GEO & 0.1 & 10 & $5 \times 10^{-2}$ \\
GC & 0.1 & 10 & $5 \times 10^{-2}$ \\
CM & 0.05 & 10 & $1 \times 10^{-4}$ \\
CEV & 0.05 & 25 & $5 \times 10^{-3}$ \\
CIP & 0.05 & 10 & $5 \times 10^{-4}$ \\
\hline
\end{tabular}

Table S2: Obtained accuracies for ML models trained on $\log \left|V_{i j}\right|$. This table contains the same models and predictions as Table 2 of the main article. The predictions are translated into absolute scale to calculate MAEs and $\left(R^{2}\right.$ values $)$. MAEs are given in $\mathbf{m e V}$.

\begin{tabular}{lcccc}
\hline & \multicolumn{2}{c}{ KRR } & \multicolumn{2}{c}{ SVR } \\
& rbf & lap & rbf & lap \\
\hline GEO & $2.45(0.36)$ & $1.75(0.71)$ & $1.90(0.72)$ & $1.46(0.82)$ \\
GC & $2.06(0.63)$ & $1.87(0.57)$ & $1.73(0.73)$ & $1.73(0.69)$ \\
CM & $2.09(0.46)$ & $1.72(0.70)$ & $2.23(0.39)$ & $1.54(0.77)$ \\
CEV & $4.25(-8.38)$ & $2.97(-1.15)$ & $5.01(-74.8)$ & $2.81(-0.57)$ \\
CIP & $1.54(0.73)$ & $1.13(0.88)$ & $1.20(0.87)$ & $1.22(0.84)$ \\
\hline
\end{tabular}


Table S3: Obtained MAEs and ( $R^{2}$ values) for KRR models trained on $\log \left|V_{i j}\right|$. Comparing the errors on the training set (MD15k) and the test set (MDTest) shows large differences for models using the Laplacian kernel. This is an indication for overfitting. The tendency to overfit also depends on the choice of features but it is larger for the Laplacian kernel for all features we investigated. This suggests that the Laplacian kernel is more prone to overfitting and therefore not ideally suited for the problem at hand.

\begin{tabular}{lcccc}
\hline & \multicolumn{2}{c}{ KRR (rbf) } & \multicolumn{2}{c}{ KRR (lap) } \\
& MD15k & MDTest & MD15k & MDTest \\
\hline GEO & $0.54(0.93)$ & $0.55(0.92)$ & $0.44(0.95)$ & $0.51(0.93)$ \\
GC & $0.45(0.95)$ & $0.46(0.95)$ & $0.39(0.96)$ & $0.50(0.93)$ \\
CM & $0.34(0.97)$ & $0.47(0.94)$ & $0.20(0.99)$ & $0.42(0.96)$ \\
CEV & $0.49(0.93)$ & $0.68(0.87)$ & $0.31(0.97)$ & $0.61(0.90)$ \\
CIP & $0.36(0.97)$ & $0.37(0.96)$ & $0.19(0.99)$ & $0.35(0.97)$ \\
\hline
\end{tabular}
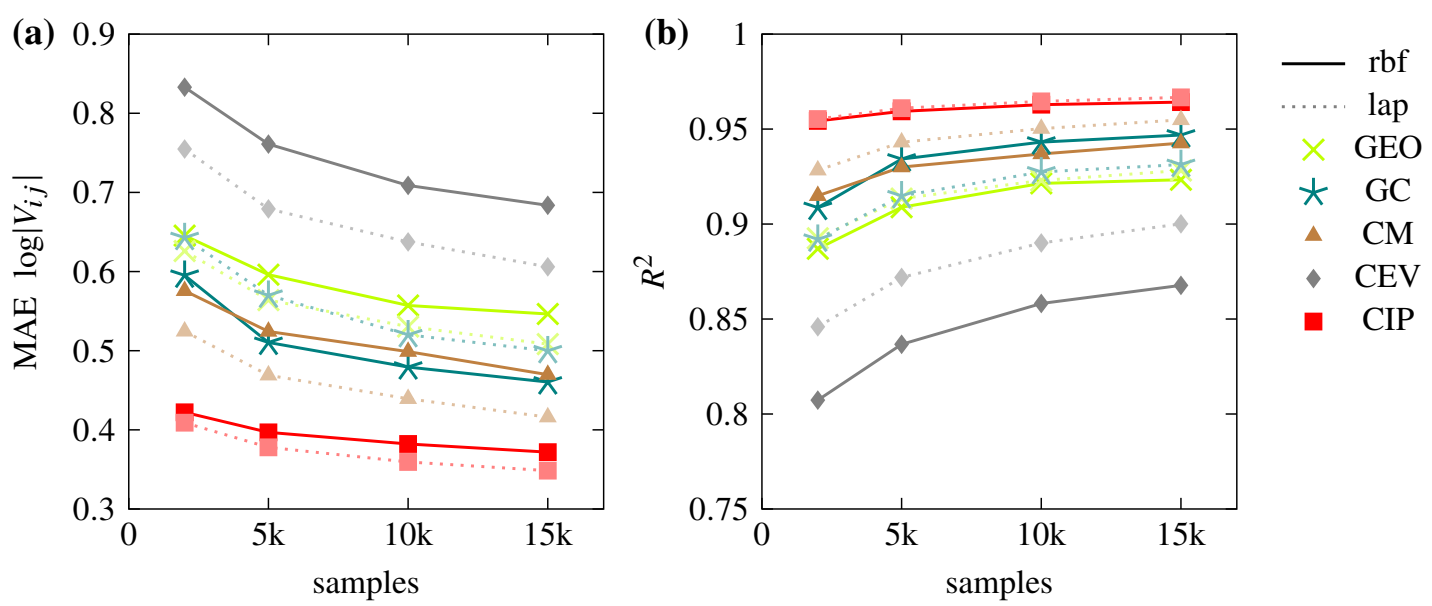

Figure S7: Learning curves of KRR machine learning models with Gaussian kernel (solid lines) and Laplacian kernel (dotted lines) for different features. 


\section{Mobility Anisotropy}

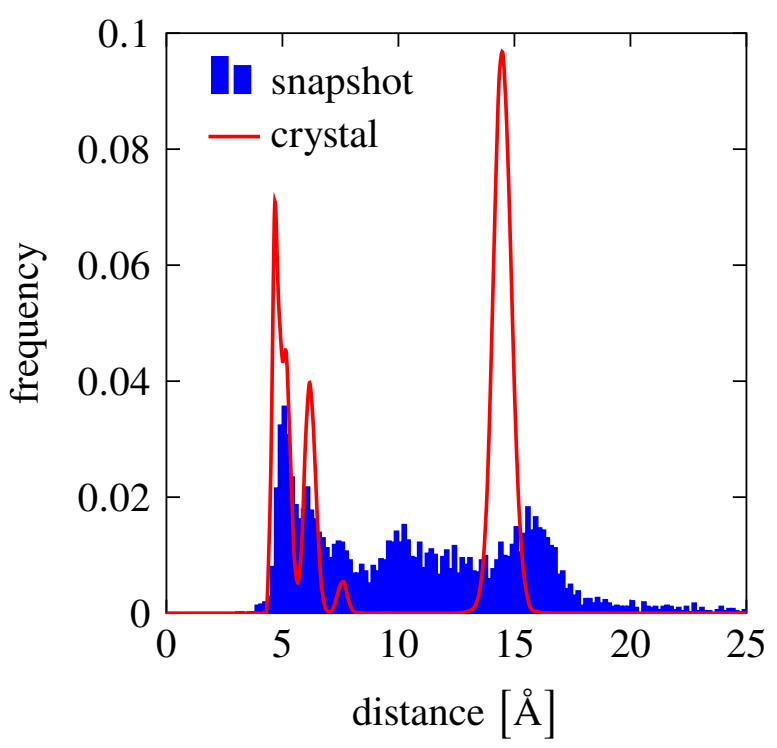

Figure S8: Dimer distance distribution of the MD snapshot used for kMC simulations (blue) and a pentacene crystal at room temperature from our previous work (red). ${ }^{1}$ 


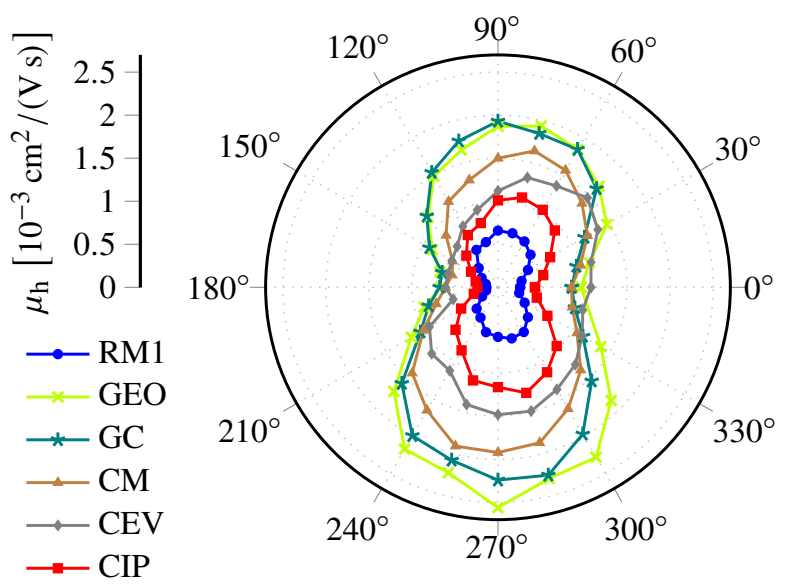

Figure S9: Mobility anisotropy obtained with $V_{i j}$ predictions from KRR models with Gaussian kernel trained on $\left|V_{i j}\right|$. All simulations are obtained using the off-lattice kMC method.

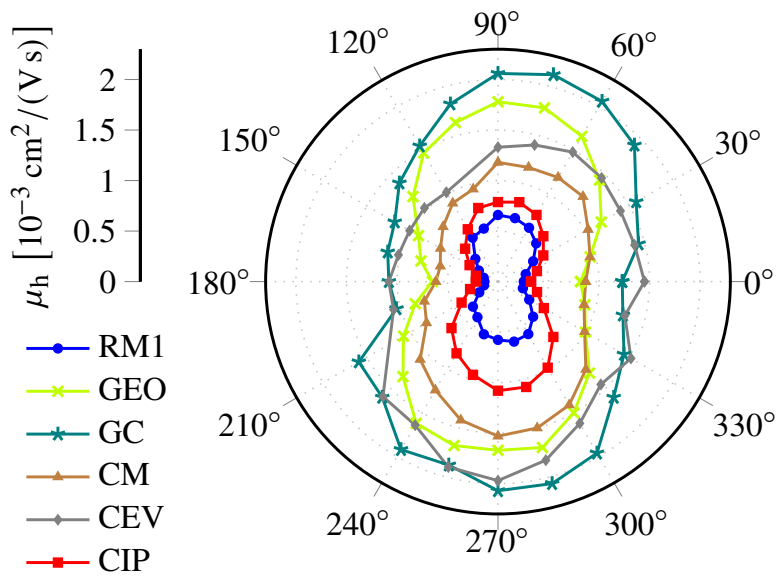

Figure S10: Mobility anisotropy obtained with $V_{i j}$ predictions from KRR models with Laplacian kernel trained on $\left|V_{i j}\right|$. All simulations are obtained using the off-lattice kMC method. 

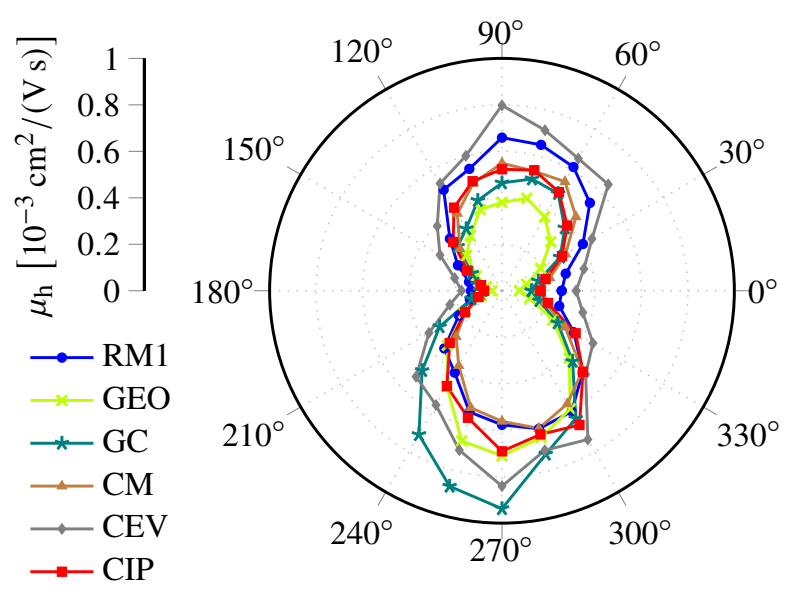

Figure S11: Mobility anisotropy obtained with $V_{i j}$ predictions from KRR models with Laplacian kernel trained on $\log \left|V_{i j}\right|$. All simulations are obtained using the off-lattice kMC method.

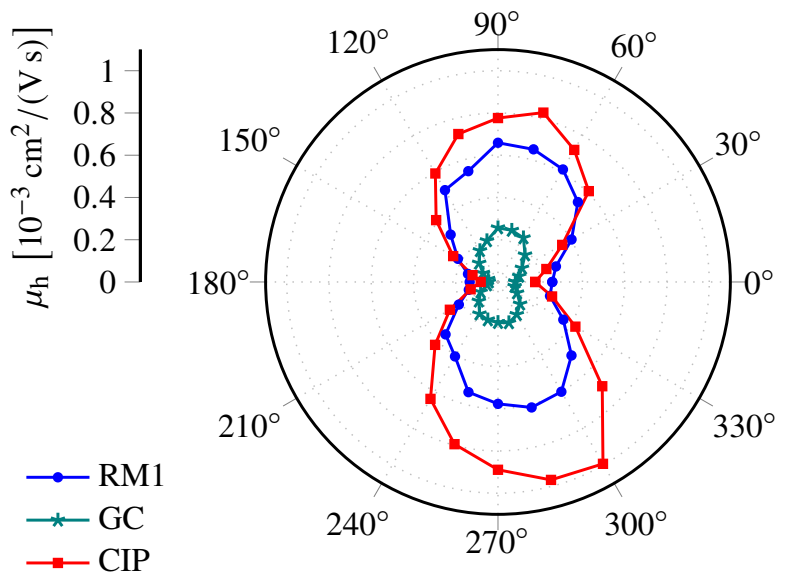

Figure S12: Mobility anisotropy obtained with $V_{i j}$ predictions from KRR models with Laplacian kernel trained with the SP15k dataset and trained on $\log \left|V_{i j}\right|$ target. All simulations are obtained using the off-lattice kMC method. 

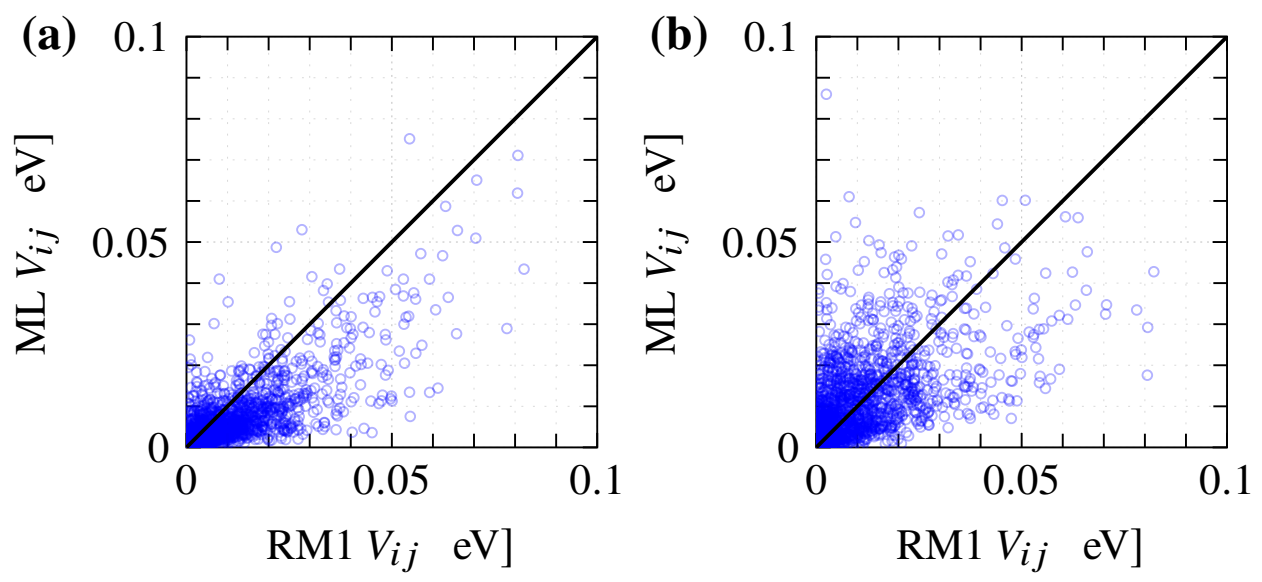

Figure S13: Accuracy of ML predictions versus semi-empirical RM1 simulation using KRR models with Gaussian kernel function and (a) CIP feature, (b) GC feature. The models were trained on $\log \left|V_{i j}\right|$ with the SP15k dataset and used to predict the MDTest dataset. 


\section{E Transferability of the ML Framework}

Table S4: Comparison of ML models using a combined training set of pentacene and DNTT molecules. MAE and ( $R^{2}$ values) are obtained with KRR models using rbf kernels and GC features trained on $\log \left|V_{i j}\right|$.

\begin{tabular}{cc|ccc}
\hline \multicolumn{2}{c}{ Training data } & \multicolumn{3}{|c}{ Test data } \\
Pentacene & DNTT & Pentacene & DNTT & Combined \\
\hline $15 \mathrm{k}$ & - & $0.46(0.95)$ & $1.17(0.59)$ & - \\
$10 \mathrm{k}$ & $5 \mathrm{k}$ & $0.46(0.95)$ & $0.55(0.93)$ & $0.49(0.94)$ \\
$5 \mathrm{k}$ & $10 \mathrm{k}$ & $0.48(0.94)$ & $0.52(0.93)$ & $0.49(0.94)$ \\
- & $15 \mathrm{k}$ & $0.69(0.88)$ & $0.51(0.94)$ & - \\
\hline $5 \mathrm{k}$ & $5 \mathrm{k}$ & $0.51(0.94)$ & $0.55(0.93)$ & $0.50(0.94)$ \\
$10 \mathrm{k}$ & $10 \mathrm{k}$ & $0.46(0.95)$ & $0.53(0.93)$ & $0.48(0.94)$ \\
$15 \mathrm{k}$ & $15 \mathrm{k}$ & $0.45(0.95)$ & $0.52(0.93)$ & $0.47(0.95)$ \\
\hline
\end{tabular}

Table S5: Comparison of ML models using a combined training set of pentacene and DNTT molecules. MAE and ( $R^{2}$ values) are obtained with KRR models using rbf kernels and CM features trained on $\log \left|V_{i j}\right|$.

\begin{tabular}{cc|ccc}
\hline \multicolumn{2}{c|}{ Training data } & \multicolumn{3}{|c}{ Test data } \\
Pentacene & DNTT & Pentacene & DNTT & Combined \\
\hline $15 \mathrm{k}$ & - & $0.47(0.94)$ & $4.71(-3.24)$ & - \\
$10 \mathrm{k}$ & $5 \mathrm{k}$ & $0.47(0.94)$ & $0.59(0.91)$ & $0.52(0.93)$ \\
$5 \mathrm{k}$ & $10 \mathrm{k}$ & $0.49(0.94)$ & $0.57(0.91)$ & $0.52(0.93)$ \\
- & $15 \mathrm{k}$ & $4.80(-3.15)$ & $0.58(0.91)$ & - \\
\hline $5 \mathrm{k}$ & $5 \mathrm{k}$ & $0.50(0.94)$ & $0.60(0.90)$ & $0.54(0.92)$ \\
$10 \mathrm{k}$ & $10 \mathrm{k}$ & $0.47(0.94)$ & $0.57(0.91)$ & $0.51(0.93)$ \\
$15 \mathrm{k}$ & $15 \mathrm{k}$ & $0.46(0.95)$ & $0.55(0.92)$ & $0.50(0.93)$ \\
\hline
\end{tabular}




\section{References}

(1) Lederer, J.; Kaiser, W.; Mattoni, A.; Gagliardi, A. Machine Learning-Based Charge Transport Computation for Pentacene. Adv. Theory Simul. 2019, 2, 1800136. 